\title{
HOLOGRAPHIE NUMÉRIQUE pour la photostimulation
de circuits neuronaux
}

\author{
Emiliano RONZITTII ${ }^{1}$, Dimitrii TANESE ${ }^{1}$, Alexis PICOT $^{1}$, Benoît C. FORGET ${ }^{1}$, Valentina EMILIANI ${ }^{1}$, \\ Eirini PAPAGIAKOUMOU \\ ${ }^{1}$ Wavefront-Engineering Microscopy group, Neurophotonics Laboratory, CNRS UMR8250, Université Paris Descartes, \\ 45 rue des Saints-Pères, 75270 Paris Cedex 06, France \\ ${ }^{2}$ Institut national de la santé et de la recherche médicale - Inserm \\ benoit.forget@parisdescartes.fr
}

Le développement de techniques originales de manipulation de la lumière ont permis de grandes avancées dans le domaine de l'optogénétique. Elles permettent d'étudier et de stimuler la communication au sein des circuits neuronaux et du cerveau avec une précision spatiale et temporelle correspondant à l'activation d'une cellule unique au sein d'un circuit.

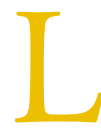
e contrôle de l'activation de circuits neuronaux permettrait de comprendre et de réguler le fonctionnement du cerveau, dans son état de santé ou de pathologie. Pour déterminer le rôle de chacun de ces circuits au sein du cerveau dans sa globalité et afin de réduire la complexité de cette tâche, l'approche habituelle consiste à les décomposer en sous-ensembles et à moduler sélectivement leur activité. L'optogénétique permet aujourd'hui d'envisager un tel contrôle coordonné grâce d'une part au développement continu des sondes, ou actionneurs, c'est-à-dire des protéines photosensibles (opsines, voir figure 1) et d'autre part à la mise en œuvre de techniques et de stratégies d'illumination idoines.

L'illumination en champ large est la première méthode à avoir été mise en œuvre pour activer les sondes optogénétiques, et elle continue d'être largement utilisée pour l'étude des circuits neuronaux à la fois in vitro et in vivo. Dans cette approche le ciblage d'une population choisie de neurones résulte du marquage génétique, et la résolution temporelle est fixée par la dynamique des canaux ioniques et par les propriétés des cellules marquées (niveau de l'expression de l'opsine, potentiel membranaire...).
Cette approche est aussi limitée du fait que l'excitation est simultanée pour l'ensemble des cellules génétiquement marquées. Elle ne permet donc pas un contrôle spatial et temporel de l'activation à l'échelle de la cellule unique, nécessaire pour reproduire ou mimer le fonctionnement physiologique des circuits neuronaux. Une meilleure résolution spatiale peut être obtenue en utilisant l'absorption non linéaire (à 2 photons, 2P) ce qui par ailleurs permet une pénétration plus profonde dans les tissus grâce au remplacement de la source de lumière visible par une source proche infrarouge. Toutefois, la faible conductance des actionneurs photosensibles (canaux ou pompes ioniques), tels que la channelrhodopsine, ainsi que leur très faible nombre dans le volume d'excitation, qui est de l'ordre du femtolitre (correspondant à un faisceau $2 \mathrm{P}$ focalisé), rendent difficile la création de courants transmembranaires suffisants pour conduire un neurone au seuil de déclenchement $\mathrm{du}$ potentiel d'action.

Ce challenge a conduit au développement de nouvelles approches de photostimulation 2P. Celles-ci peuvent être regroupées en deux catégories : parallèle ou séquentielle. Les techniques séquentielles consistent à balayer un faisceau laser de façon
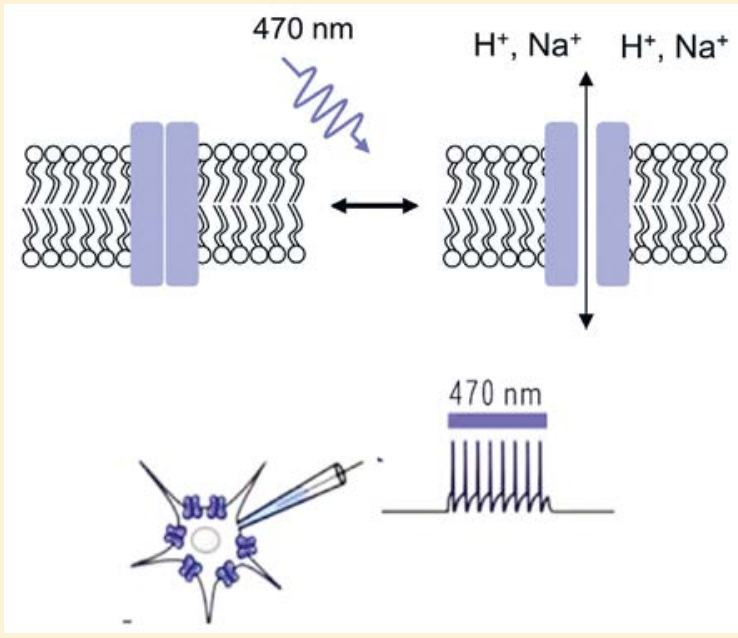

Figure 1. Les channelrhodopsines sont les sondes optogénétiques les plus utilisées. Elles permettent de dépolariser des neurones rapidement en faisant circuler des cations à travers la membrane en présence de lumière bleue. 
à couvrir la surface d'une cellule (ou plusieurs cellules consécutivement). Les approches parallèles permettent d'illuminer simultanément toute la surface d'une ou plusieurs cellules en utilisant un objectif à faible ouverture numérique et une technique de mise en forme du faisceau comme l'holographie numérique (computergenerated holography, CGH). Dans cet article nous nous concentrerons spécifiquement sur l'application de l'holographie numérique à l'étude et au contrôle des circuits neuronaux.

\section{Holographie mumérique}

Développée à l'origine pour la réalisation de pièges optiques multiples, la technique d'holographie numérique consiste à calculer, à l'aide d'un algorithme itératif basé sur la transformée de Fourier (IFTA), la figure d'interférence ou l'hologramme de phase formé par la rétropropagation de la répartition d'intensité lumineuse souhaitée (forme ou image cible) dans le plan focal de l'objectif vers le plan de diffraction. Cet hologramme de phase est numérisé et envoyé à un modulateur spatial de lumière à base de cristaux liquides (liquid-crystal spatial light modulator, LC-SLM) placé dans ce plan. Chaque pixel de la matrice de cristaux liquides impose une modulation de phase locale, contrôlée par la tension qui lui est appliquée. Ainsi l'hologramme calculé numériquement devient un masque de phase pixélisé dont l'illumination par un faisceau laser (faisceau de référence) créera dans le plan focal une distribution lumineuse conforme à l'image cible. Cette image cible peut être une distribution arbitraire en deux ou trois dimensions, allant de faisceaux limités par la diffraction («spots») à des formes arbitraires de plus grande dimension. Pour l'application à l'étude et au contrôle de l'activité neuronale nous avons proposé il y a quelques années de créer l'hologramme de phase à partir d'une image
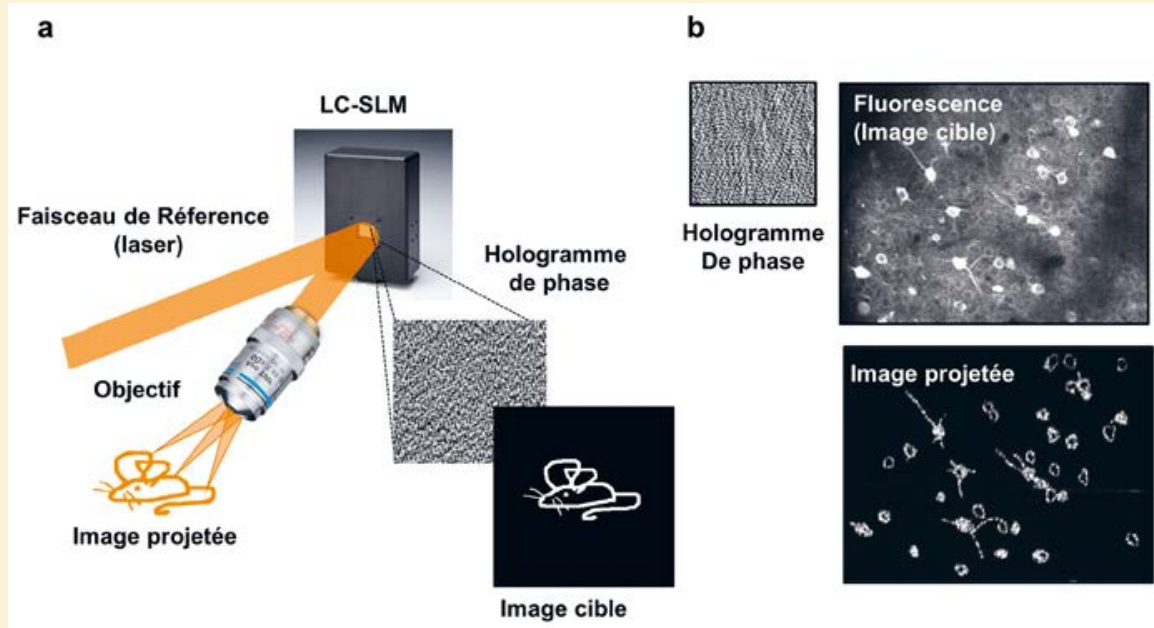

Figure 2. Holographie numérique (computer generated holography, CGH). (a) En CGH une distribution arbitraire (image cible) sert d'entrée à un algorithme itératif basé sur la transformée de Fourier. Celui-ci calculera la figure d'interférence ou hologramme de phase qui, une fois illuminée par le faisceau de référence (laser), reproduira cette distribution arbitraire dans le plan focal de l'objectif (image). (b) Pour la photostimulation de neurones, on utilise une image de fluorescence en tant qu'image cible.

de fluorescence de la structure neuronale à photostimuler [1]. Une fois enregistrée, cette image de fluorescence sert à « dessiner » la répartition de l'intensité lumineuse qui sera idéalement ajustée à cette structure (voir figure 2).

\section{Champ d'excitation, imhomogénéité et speckle}

En CGH, le nombre et la taille des pixels de la matrice LC-SLM déterminent l'extension maximale du champ d'excitation (field of excitation, FOE) et aussi l'efficacité de diffraction, définie comme le rapport de l'intensité dans le champ diffracté par rapport à celle du faisceau incident. Avec les LCSLM actuels il est possible d'obtenir expérimentalement une efficacité de $95 \%$ au centre et de $38 \%$ aux bords du champ de vue, ce qui correspond à peu près à la limite théorique. Outre le problème de cette inhomogénéité en tant que telle $e^{1}$ se pose aussi celui du terme non diffracté, dit terme d'ordre zéro, qui crée une tache focalisée au centre du FOE où se concentre toute l'intensité lumineuse non diffractée qui peut atteindre jusqu'à $25 \%$ de l'intensité totale, selon le profil de phase appliqué au LC-SLM. Il est possible de l'éliminer en plaçant un cache ou un diaphragme dans un plan conjugué au plan image. Une autre approche sans diaphragme (et donc sans limitation du FOE) consiste à utiliser une lentille cylindrique pour étirer le terme d'ordre zéro sur une ligne et donc de diminuer fortement son intensité, spécialement en excitation 2P. Dans cette configuration, l'hologramme devra compenser l'effet de cette lentille cylindrique pour restituer la forme désirée.

La qualité des formes arbitraires créées par CGH est aussi limitée par le speckle (tavelures). Cette limitation inhérente à l'utilisation de l'IFTA est due à la présence de discontinuités de phase dans le plan image. Les fluctuations relatives d'intensité dues au speckle atteignent jusqu'à $20 \%$ pour des excitations $1 \mathrm{P}$ et $50 \%$ pour les excitations $2 \mathrm{P}$. Il est possible de les réduire en effectuant une moyenne temporelle à l'aide d'un diffuseur en rotation ou en projetant une séquence de plusieurs hologrammes équivalents. Un profil plus lisse peut être obtenu en introduisant des modifications ad hoc à l'algorithme afin de retirer les singularités en

Que l'on peut pallier par exemple en utilisant une image de départ normalisée afin de projeter une intensité a priori plus importante vers les endroits du FOE où l'efficacité de diffraction est plus faible et vice versa, ou en projetant plusieurs spots hors du FOE en ajustant leur intensité ou leur taille en fonction de la position du spot d'intérêt. 
spirale dans le masque de phase. Par ailleurs, il a été montré que la méthode dite de contraste de phase généralisé (generalized phase contrast, GPC) permet de créer des formes arbitraires en $2 \mathrm{D}$ sans speckle de dimensions suffisantes pour la photostimulation d'une population choisie de neurones au sein du réseau. Récemment, des équipes de recherches ont montré que la technique GPC peut être adaptée pour former des répartitions de lumière en $3 \mathrm{D}$ en la combinant avec la technique CGH.

\section{Résolution spatialle}

En plus de la résolution spatiale telle que définie en microscopie optique par la fréquence spatiale maximale transmise par l'objectif (donc liée à son ouverture numérique), il convient de définir celle de précision de localisation, c'est-à-dire la précision avec laquelle on peut atteindre un point
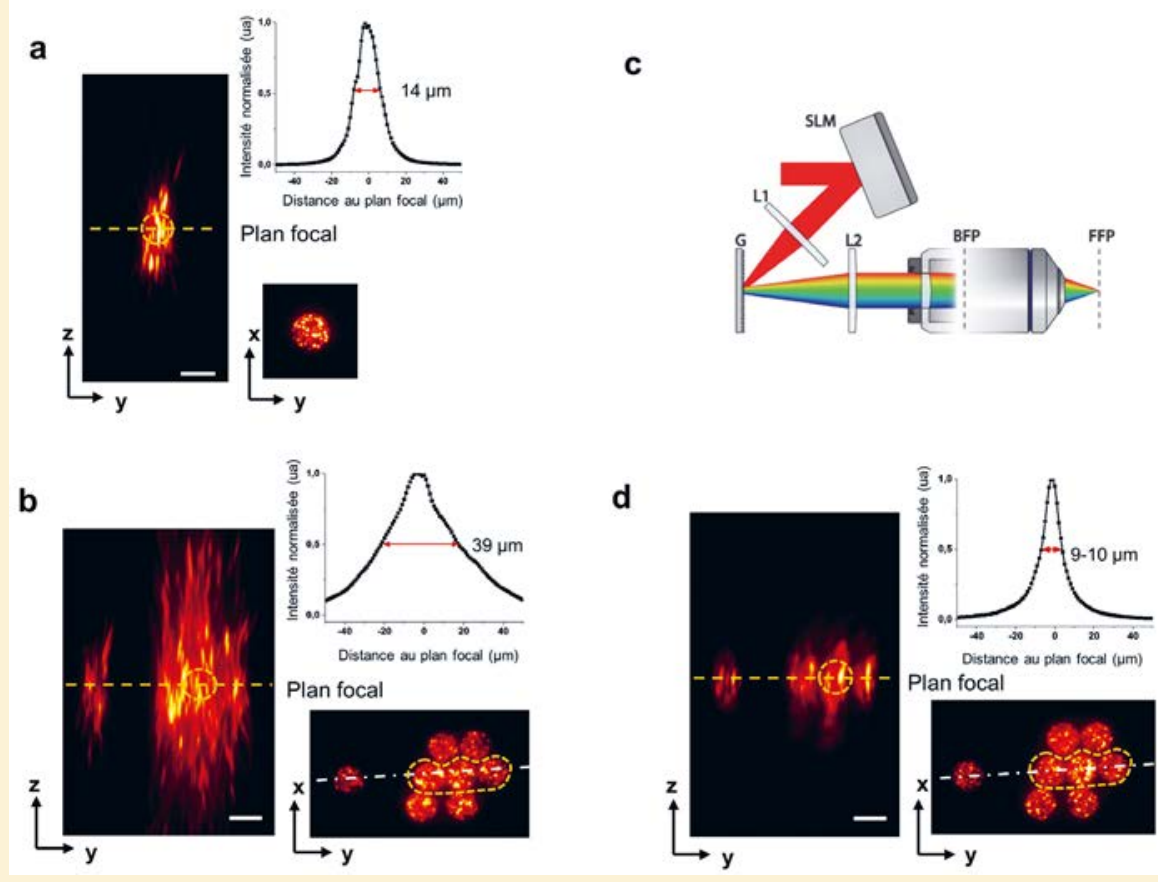

Figure 3. Holographie numérique (CGH) avec focalisation temporelle (temporal focusing, TF). (a)-(b) Mesures expérimentales de la distribution d'intensité dans les plans perpendiculaires (xy) et parallèle (yz) à la direction de propagation, pour un seul (a) et plusieurs (b) « spots » de $10 \mu \mathrm{m}$ de diamètre créés par CGH sans TF. Barre d'échelle 10 $\mu \mathrm{m}$. Les cercles (trait jaune tireté) indiquent l'ordre de grandeur de la taille du soma (ou corps cellulaire) d'une cellule. Les profils d'intensité tracés montrent que leur largeur à mi-hauteur dépend de la dimension latérale de la forme projetée, $14 \mu \mathrm{m}$ pour un diamètre de $10 \mu \mathrm{m}$ contre $39 \mu \mathrm{m}$ pour un diamètre d'environ $30 \mu \mathrm{m}$. (c) Dispositif expérimental couplant CGH et TF. G : réseau de diffraction; L1,L2 : lentilles; BFP : plan focal arrière ; FFP : plan focal avant. (d) Mesure expérimentale de la distribution d'intensité pour la même forme qu'en (b), cette fois avec TF. La largeur à mi-hauteur est considérablement diminuée et devient indépendante de la forme projetée. contenu fréquentiel d'une impulsion ultrabrève. Chacune des fréquences du spectre se propage vers le plan focal avec un angle différent. Ainsi l'impulsion est étirée au-dessus et au-dessous du plan focal, et il n'y a que dans ce plan focal que l'intensité est suffisante pour une excitation 2P efficace (voir figure 3). Il est ainsi possible de réaliser une photostimulation $2 \mathrm{P}$ à l'échelle cellulaire avec une résolution temporelle de la milliseconde à la fois in vitro et in vivo.

\section{Profondeutir de pénétration et photostimulation 3D}

En plus de permettre une résolution axiale de l'ordre du micron, la focalisation temporelle augmente la robustesse à la diffusion lors de la propagation de l'impulsion lumineuse dans le milieu biologique. En effet chaque composante fréquentielle de l'impulsion suit un trajet différent à travers ce milieu hétérogène pour se recombiner dans le plan focal. En pratique ceci diminue la probabilité d'interférence entre les photons diffusés et les photons balistiques, et permet de projeter la forme souhaitée jusqu'à une profondeur de deux fois la longueur de diffusion.

Si l'holographie numérique permet bien le positionnement en 3D des formes arbitraires, utilisé par exemple pour la photostimulation optogénétique $2 \mathrm{P}$ par scanning en spirale, l'approche 3D classique n'est pas compatible avec le temporal focusing TF puisque les différents plans ne peuvent être imagés simultanément sur le réseau de diffraction. Pour surmonter cette difficulté nous avons récemment développé un nouveau système basé sur l'utilisation de deux SLMs [3]. Le premier (voir figure 4) permet de créer la forme arbitraire dont la résolution axiale sera obtenue grâce au TF. Les composantes fréquentielles dispersées par le réseau sont modulées par le second SLM auquel sont adressées une ou plusieurs lentilles de Fresnel, et qui ainsi projette la forme vers la position en 3D associée à chacune d'elles. 
a

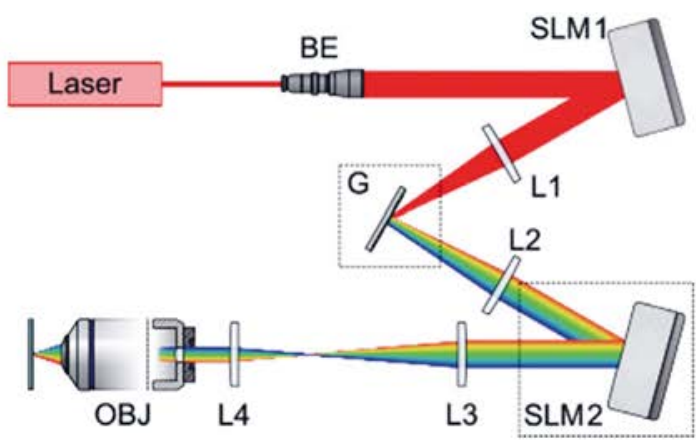

b

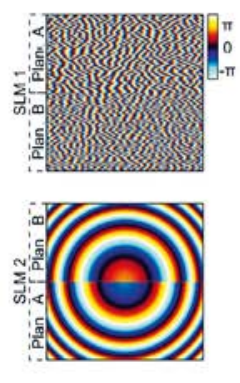

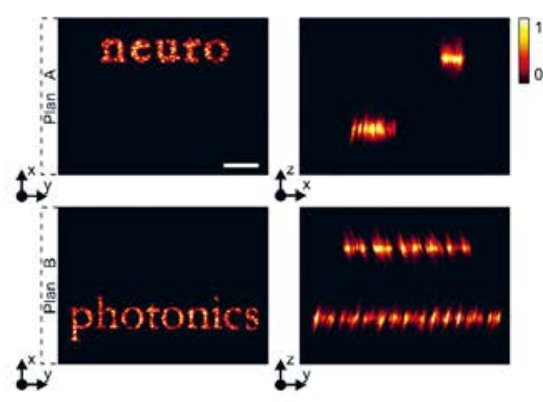

Figure 4. Photostimulation 3D. (a) Dispositif expérimental couplant CGH et TF pour la photostimulation 3D. BE : expanseur de faisceau ; $\mathrm{G}$ : réseau de diffraction; L1,L2,L3,L4 : lentilles; OBJ1 : objectif de microscope. (b) En haut à gauche les hologrammes envoyés au SLM1 correspondant aux mots « neuro » (plan A) et « photonics » (plan B). En bas à gauche les lentilles de Fresnel envoyées au SLM2 pour projeter ces deux respectivement dans le plan A $(20 \mu \mathrm{m}$ au-dessus du plan focal) et B $(20 \mu \mathrm{m}$ au-dessous). Au centre, les images de fluorescence obtenues dans les plans A (en haut) et B (en bas). À droite la projection dans deux plans distincts et orthogonaux à l'axe de propagation.

\section{Résolution temporelle}

Les techniques parallèles permettent également une excitation efficace temporellement puisque le photocourant induit est intégré sur l'ensemble de la surface de la cellule couverte par l'illumination. Il est donc possible d'utiliser des impulsions de plus courte durée. Cette durée doit être étudiée en fonction de la dynamique propre à chaque opsine, rapide ou lente. En pratique, il a été montré qu'il est possible de déclencher un train de potentiels d'actions avec une résolution de l'ordre de la milliseconde et une précision sub-miliseconde, et ce à des profondeurs de l'ordre de $100 \mu \mathrm{m}$ et avec des intensités de l'ordre $1 \mathrm{~mW} / \mu^{2}$ ou $100 \mu \mathrm{W} / \mu \mathrm{m}^{2}$.

\section{Perspectives}

La possibilité de déclencher des potentiels d'actions de façon contrôlée et en parallèle pour plusieurs neurones dans un espace 3D permet d'envisager concrètement la modulation et l'étude de réseaux neuronaux. L'intensité nécessaire " par neurone » étant de l'ordre de $100 \mu \mathrm{W} / \mu \mathrm{m}^{2}$ ou moins permet de penser que le nombre de neurones étudiés ou stimulés ne serait pas limité par la puissance optique totale disponible (plus de $10 \mathrm{~W}$ en sortie du laser). Et, bien que certaines limitations (comme l'échauffement local des tissus) doivent encore être étudiées plus en détail, les résultats prometteurs sur des cellules isolées nous laissent prévoir à court terme leur adaptation à l'étude de l'organe entier.

\section{POUR EN SAVOIR PLUS}

[1] E. Papagiakoumou, Optical developments for optogenetics. Biol Cell 105:443 (2013)

[2] E. Papagiakoumou, V. de Sars, D. Oron, V. Emiliani, Patterned two-photon illumination by spatiotemporal shaping of ultrashort pulses. Opt Express 16:22039 (2008)

[3] O. Hernandez et al., Three-dimensional spatiotemporal focusing of holographic patterns. Nat Commun 7:11928 (2016)

\section{IDIL SPÉCIALISTE DES TECHNOLOGIES FIBRES OPTIQUES ET LASER}

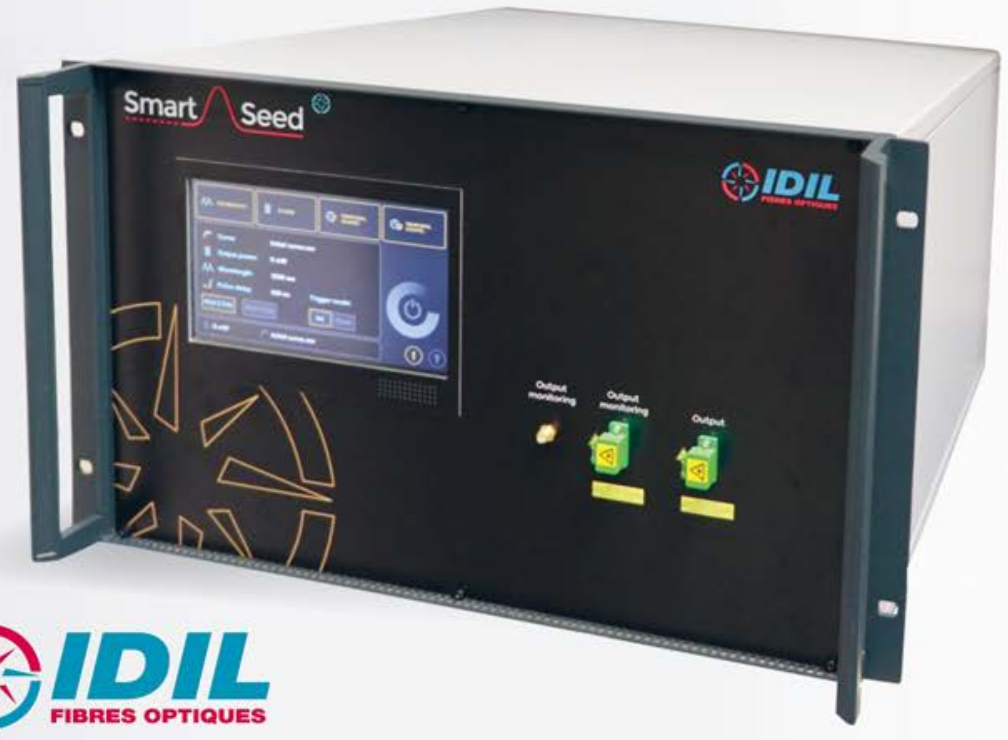

\section{Smart Seed INJECTEUR POUR LASER}

\section{HAUTE PUISSANCE}

\section{PERFORMANES}

$\lambda$ 1030, 1053, $1064 \mathrm{~nm}+$ sur mesure

Énergie > $10 \mathrm{~nJ}$ (20 ns largeur d'impulsion)

Rapport d'extinction temporelle $>50 \mathrm{~dB}$

Forme d'impulsion ajustable

Résolution temporelle 125 ps

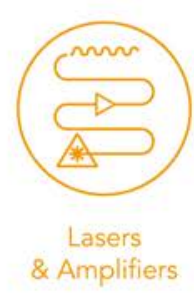

